\title{
Sudakov resummation from the BFKL evolution
}

\author{
M. A. Nefedov $\circledast^{*}$ \\ Université Paris-Saclay, CNRS, IJCLab, 91405 Orsay, France and Samara National Research University, \\ Moskovskoe Shosse, 34, 443086 Samara, Russia
}

(Received 31 May 2021; accepted 1 September 2021; published 28 September 2021)

\begin{abstract}
The leading-logarithmic (LL) gluon Sudakov form factor is derived from rapidity-ordered Balitsky-Fadin-Kuraev-Lipatov evolution with longitudinal-momentum conservation. This derivation further clarifies the relation between high-energy and transverse-momentum-dependent factorizations and can be extended beyond the LL approximation as well.
\end{abstract}

DOI: 10.1103/PhysRevD.104.054039

\section{INTRODUCTION}

The interplay between the effects of high-energy QCD and transverse-momentum-dependent (TMD) factorization has become a topic of intensive theoretical studies in recent years; see, e.g., Refs. [1-11]. Regions of applicability of these two formalisms overlap when observables related to transverse momentum $\left(q_{T}\right)$ are studied in the process containing another large-scale $M$ in hadronic or leptonhadron collision with large center-of-mass energy $\sqrt{S}$, while all three scales become hierarchical: $\sqrt{S} \gg M \gg q_{T}$. The rapidity-evolution equation for a gluon TMD parton distribution function (PDF) was conjectured few years ago $[1,2]$, which unifies the nonlinear Balitsky-Kovchegov $[12,13]$ evolution equation in the small- $x$ limit with the linear Collins-Soper-Sterman (CSS)-type evolution of TMD PDFs familiar at moderate values of $x$ (see, e.g., the monograph [14] for a review of TMD factorization). Unfortunately, the systematic study of this evolution equation is a very challenging task. Its real-emission kernel is essentially three dimensional, interpolating between the small- $x$ (Regge) limit with characteristic nontrivial transverse-momentum dynamics and moderate- $x$ limit, for which longitudinal-momentum conservation is crucial [15]. More recently, the origin of corrections $\sim\left(\alpha_{s} \ln ^{2}\left(M^{2} / q_{T}^{2}\right)\right)^{n}$, the so-called "Sudakov" double logarithms, in the approach of Refs. [1,2] were further clarified in Ref. [3] where the roles of the conformally invariant cutoff for rapidity divergences as well as of the conservation of the dominating light-cone component of momentum where emphasized. However, connection to the physics of the standard

\footnotetext{
*nefedovma@gmail.com
}

Published by the American Physical Society under the terms of the Creative Commons Attribution 4.0 International license. Further distribution of this work must maintain attribution to the author(s) and the published article's title, journal citation, and DOI. Funded by SCOAP.
Balitsky-Fadin-Kuraev-Lipatov (BFKL) [17-19] evolution equation, which is the cornerstone of high-energy QCD, seems to be lost in these recent developments.

The goal of the present paper is to restore this connection. As it will be shown below, the Sudakov suppression of the gluon unintegrated PDF (UPDF) in the limit $q_{T} \ll M$ can be reproduced starting from the standard leading-logarithmic (LL) BFKL equation if one imposes the conservation of the leading light-cone momentum component through the cascade of real emissions.

The present study has also been motivated by a few other considerations. First, it is known that resummation of higher-order corrections enhanced by $\ln M / q_{T}$ in the formalisms of soft-collinear effective theory (SCET) [20-22] and TMD factorization [14] are driven by rapidity divergences. On the other hand, the kernel of the BFKL equation can be derived as a coefficient of the rapiditydivergent part of the four-Reggeon Green's function in Lipatov's gauge-invariant effective field theory (EFT) for high-energy processes in QCD [23]; see, e.g., Ref. [24]. The gluon Regge trajectory at one [25] and two loops $[26,27]$ can be computed as the coefficient of the rapidity divergence of Reggeized gluon self-energy. These results suggest a close connection between rapidity divergences in SCET and BFKL physics; see, e.g., Refs. [28,29] for an approach to incorporate BFKL physics into SCET language.

Further motivation is that in the phenomenology of highenergy factorization, it has long been understood that the most useful phenomenological UPDFs must include effects of Sudakov resummation in addition to BFKL effects. A well-known example of such UPDF is the Kimber-Martin-Ryskin-Watt formula [30-33]. The combination of a Sudakov-improved UPDF with gauge-invariant matrix elements with Reggeized (off-shell) initial-state partons gives excellent phenomenological results for observables such as dijet correlations [34-36], multijet [37] correlations, angular correlations of pairs of $B$ [38] and 
$D$ mesons [39,40], $J / \psi$ pair production [41], Drell-Yan process [42-44], and many other observables. Derivation of a Sudakov form factor from BFKL evolution in which $t$-channel partons are Reggeized provides further evidence that such a phenomenological approach is selfconsistent.

The present paper has the following structure: In Sec. II, the high-energy factorization is reviewed, and the concept of the unintegrated PDF is introduced. In Sec. III, the modified BFKL Green's function with longitudinalmomentum conservation is computed. In Sec. IV, the Sudakov form factor for the UPDF is derived in the leading- (doubly) logarithmic approximation. In Sec. V, the same result is rederived in a heuristic way to expose more clearly its physical content, and in Sec. VI, the relations of our derivation with other results in the literature and directions for further studies are discussed. In the Appendix, we put the details of the derivation of the function $\Delta \chi(\gamma, \lambda)$, which is used for the study of the modified BFKL Green's function.

\section{HIGH-ENERGY FACTORIZATION AND UNINTEGRATED PDF}

The high-energy factorization (HEF) was originally introduced in Refs. [45-48] as a tool to resum higherorder QCD corrections $\sim \alpha_{s}^{n} \ln ^{n}(1 / z)$ in the hard-scattering coefficient of the collinear parton model (CPM). Here, variable $z$ can be, e.g., the ratio of the squared transverse mass $\left(M_{T}\right)$ of the final state, which is detected in a hard inclusive reaction of interest to the partonic center-of-mass energy $z=M_{T}^{2} / \hat{s}$. See, e.g., Refs. [49-52] and references therein for applications of this formalism to deep-inelastic scattering and Higgs boson production. However, in addition to large logarithms $\ln (1 / z)$, the Sudakov large logarithms $\ln \left(M_{T} /\left|\mathbf{p}_{T}\right|\right)$ could also be important if transverse momentum of the final state of interest $\left|\mathbf{p}_{T}\right| \ll M_{T}$. Therefore, the following slightly more general form of high-energy factorization incorporating to some extent both resummations is more useful:

$$
\begin{aligned}
d \sigma= & \int_{0}^{1} \frac{d x_{1}}{x_{1}} \int \frac{d^{2} \mathbf{q}_{T 1}}{\pi} \Phi_{i}\left(x_{1}, \mathbf{q}_{T 1}, \mu_{Y}\right) \\
& \times \int_{0}^{1} \frac{d x_{2}}{x_{2}} \int \frac{d^{2} \mathbf{q}_{T 2}}{\pi} \Phi_{j}\left(x_{2}, \mathbf{q}_{T 2}, \mu_{Y}\right) \\
& \times d \hat{\sigma}_{i j}\left(x_{1}, \mathbf{q}_{T 1}, x_{2}, \mathbf{q}_{T 2}\right)
\end{aligned}
$$

where indices $i, j=R, Q, \bar{Q}$ with $R$ denoting the Reggeized gluon and $Q(\bar{Q})$ are Reggeized (anti-) quarks, and $\hat{\sigma}_{i j}$ is the HEF coefficient function, which can be obtained for any QCD hard process using Lipatov's highenergy EFT [23]. The four-momenta $\left(q_{1,2}^{\mu}\right)$ of Reggeized partons are equal to $q_{1,2}^{\mu}=x_{1,2} P_{1,2}^{\mu}+q_{T 1,2}^{\mu}$, where $P_{1,2}$ are four-momenta of colliding hadrons. The UPDFs $\Phi_{i}$ in Eq. (1) depend on the rapidity scale $\mu_{Y} \sim M_{T}$. The definition of $\mu_{Y}$ is

$$
\mu_{Y}=x_{1} P_{1}^{+} e^{-Y_{\mu}}=x_{2} P_{2}^{-} e^{Y_{\mu}},
$$

where $Y_{\mu}$ is the rapidity of the hard final state of $d \hat{\sigma}$ and light-cone components are introduced in the centerof-mass frame of $P_{1,2}$ as $P_{1}^{+}=P_{1}^{0}+P_{1}^{3}, P_{2}^{-}=P_{2}^{0}-P_{2}^{3}$. The gluon UPDF in pure gluodynamics (QCD with $n_{F}=0$ ) will be the main object of our present study. In the purely perturbative approach, ignoring both the nonperturbative content in the TMD $\left(\left|\mathbf{p}_{T}\right| \ll M_{T}\right)$ region as well as violations of collinear factorization due to the genuine gluon-saturation effects, and in the leadinglogarithmic approximation in the BFKL sense, which is detailed below, the UPDF is related to the collinear momentum-density $\operatorname{PDF} \tilde{f}_{g}\left(x, \mu_{F}\right)=x f_{g}\left(x, \mu_{F}\right)$ as follows:

$\Phi_{R}\left(x, \mathbf{q}_{T}^{2}, \mu_{Y}\right)=\int_{x}^{1} \frac{d z}{z} \mathcal{C}\left(z, \mathbf{q}_{T}^{2}, \mu_{Y}, \mu_{F}\right) \tilde{f}_{g}\left(\frac{x}{z}, \mu_{F}\right)$,

where $\mathcal{C}$ is the resummation factor and $\mu_{F}$ is the factorization scale. The $\mu_{F}$ dependence should cancel out in Eq. (3) if the DGLAP evolution for PDFs is taken in an approximation consistent with the approximation for $\mathcal{C}$.

For definiteness, let us talk about the UPDF $\Phi_{R}\left(x_{1}, \mathbf{q}_{T 1}, \mu_{Y}\right)$ which is associated with partons flying in the positive rapidity direction. The factor $\mathcal{C}$ in this UPDF resums QCD corrections enhanced by rapidity gap $Y$ between the most forward gluon emission belonging to the hard-scattering coefficient of CPM, let us call it the rebounded gluon, and $Y_{\mu}$ of observed particles. The leading-logarithmic approximation for HEF resums terms $\sim\left(\alpha_{s} Y^{2}\right)^{n}$ and $\sim\left(\alpha_{s} Y\right)^{n}$ to all orders in $\alpha_{s}$ using the LL BFKL evolution equation, and such a resummation is correct up to terms suppressed as $e^{-Y}$. The standard version of HEF [47,48] uses the approximation $Y \sim \ln (1 / z)$, which is too restrictive and misses a lot of interesting physics, as we will see below. Instead, we put $Y=y-Y_{\mu}$, where $y$ is the rapidity of the rebounded gluon and introduce the collinearly unsubtracted resummation factor as (see also Fig. 1 for more insight into the notation)

$$
\begin{aligned}
\tilde{\mathcal{C}}\left(z, \mathbf{q}_{T}^{2}, \mu_{Y} \mid \mathbf{p}_{T}^{2}\right)= & \delta(z-1) \delta\left(\mathbf{q}_{T}^{2}-\mathbf{p}_{T}^{2}\right) \\
& +\hat{\alpha}_{s} \int_{Y_{\mu}}^{+\infty} d y \int \frac{d^{2} \mathbf{k}_{T}}{\pi \mathbf{k}_{T}^{2}} G\left(\mathbf{q}_{T}^{2}, z p_{+} \mid y\right. \\
& \left.-Y_{\mu},\left(\mathbf{p}_{T}-\mathbf{k}_{T}\right)^{2}, p_{+}-k_{+}\right),
\end{aligned}
$$

where $\hat{\alpha}_{s}=\alpha_{s} C_{A} / \pi, G$ is the BFKL Green's function, $p_{+}=P_{1}^{+} x_{1} / z$ is the large $(+)$ momentum component 
entering the full CPM hard process from the collinear $\mathrm{PDF}$, and $k_{+}=\left|\mathbf{k}_{T}\right| e^{y}$ is the (+) light-cone momentum component of the rebounded gluon. The factor $\hat{\alpha}_{s} / \mathbf{k}_{T}^{2}$ is the square of Lipatov's vertex for emission of the rebounded gluon, and in Eq. (4) we integrate over its phase space parametrized by rapidity $y$ and transverse momentum $\mathbf{k}_{T}$. Traditionally (see, e.g., the textbook [53]), the BFKL Green's function depends only on the rapidity interval $Y$ and transverse momenta of "incoming" $\left(\mathbf{p}_{T}-\mathbf{k}_{T}\right)$ and "outgoing" $\left(\mathbf{q}_{T}\right)$ Reggeized gluons. In our case, rapidity $Y$ does not uniquely define the longitudinal momentum of partons, and therefore, $G$ additionally depends on the $(+)$ momentum component of the $t$ channel parton entering $\left(p_{+}-k_{+}\right)$and leaving $\left(z p_{+}\right)$the Green's function. The evolution for $G$ with longitudinalmomentum dependence is described in Sec. III.

To regularize initial-state collinear divergences in $\tilde{\mathcal{C}}$, one introduces nonzero transverse momentum $\mathbf{p}_{T}$ for the incoming gluon in Eq. (4). Usually, the dimensional regularization is used for this purpose in higher-order calculations in CPM, but we find the regularization scheme with a separate regulator for collinear divergences to be more convenient for BFKL-type calculations. Eventually, we are interested in the CPM limit $\mathbf{p}_{T} \rightarrow 0$ for $\tilde{\mathcal{C}}$. If one passes to the Mellin space for $z$ dependence and $\mathbf{x}_{T}$ space for $\mathbf{q}_{T}$ dependence,

$$
\begin{aligned}
\tilde{\mathcal{C}} & \left(N, \mathbf{x}_{T}^{2}, \mu_{Y} \mid \mathbf{p}_{T}^{2}\right) \\
& =\int_{0}^{1} d z z^{N-1} \int d^{2} \mathbf{q}_{T} e^{i \mathbf{x}_{T} \mathbf{q}_{T}} \tilde{\mathcal{C}}\left(z, \mathbf{q}_{T}^{2}, \mu_{Y} \mid \mathbf{p}_{T}^{2}\right),
\end{aligned}
$$

then for $\mathbf{p}_{T} \rightarrow 0$ the collinear divergences will factorize as

$$
\begin{aligned}
\tilde{\mathcal{C}}\left(N, \mathbf{x}_{T}^{2}, \mu_{Y} \mid \mathbf{p}_{T}^{2}\right) & =Z_{\text {coll. }}\left(N, \mathbf{p}_{T}^{2}\right) \mathcal{C}\left(N, \mathbf{x}_{T}^{2}, \mu_{Y}, \mu_{F}\right), \\
Z_{\text {coll. }}\left(N, \mathbf{p}_{T}^{2}\right) & =\exp \left[-\gamma_{g g}\left(N, \hat{\alpha}_{s}\right) \ln \frac{\mathbf{p}_{T}^{2}}{\mu_{F}^{2}}\right]
\end{aligned}
$$

where $\gamma_{g g}\left(N, \hat{\alpha}_{s}\right)$ is the DGLAP anomalous dimension, and $\mathcal{C}$ is the collinearly subtracted resummation function we had been looking for. For example, if one is interested only in Leading Logarithmic Approximation (LLA) resummation of corrections enhanced by $\ln (1 / z)$, then one can ignore the complications related to longitudinal-momentum conservation and scale $\mu_{Y}$ and obtain the result

$$
\begin{aligned}
& \mathcal{C}_{\text {LLA } \ln 1 / z}\left(N, \mathbf{x}_{T}^{2}, \mu_{F}\right) \\
& \quad=\left(1+O\left(\hat{\alpha}_{s}^{3}\right)\right) \exp \left[-\gamma_{g g}\left(N, \hat{\alpha}_{s}\right) \ln \left(\mu_{F}^{2} \overline{\mathbf{x}}_{T}^{2}\right)\right],
\end{aligned}
$$

with $\overline{\mathbf{x}}_{T}=\mathbf{x}_{T} e^{\gamma_{E}} / 2$ and $\gamma_{g g}$ determined implicitly by the famous equation [54]

$$
\frac{\hat{\alpha}_{s}}{N} \chi\left(\gamma_{g g}\left(N, \hat{\alpha}_{s}\right)\right)=1,
$$

where

$$
\chi(\gamma)=2 \psi(1)-\psi(\gamma)-\psi(1-\gamma)
$$

is Lipatov's characteristic function and $\psi(\gamma)=\Gamma^{\prime}(\gamma) / \Gamma(\gamma)$. However, collinear divergences arise from terms $\sim\left(\hat{\alpha}_{s} Y\right)^{n}$, while in the present paper we are interested in effects $\sim\left(\hat{\alpha}_{s} Y^{2}\right)^{n}$. With this accuracy, collinear divergences do not appear, and $\tilde{\mathcal{C}}$ is finite in the limit $\mathbf{p}_{T} \rightarrow 0$ as it will be shown in Sec. IV.

\section{BFKL CASCADE WITH LONGITUDINAL- MOMENTUM CONSERVATION}

In the order $O\left(\alpha_{s}^{n}\right)$, the BFKL Green's function contains up to $n$ emissions or real gluons with $(+)$ momentum components $k_{1}^{+}, \ldots, k_{n}^{+}$given by $k_{i}^{+}=\left|\mathbf{k}_{T i}\right| e^{y_{i}}$ (see again Fig. 1). The overall (+) momentum-component conservation in Eq. (4) can be expressed by the delta function, which is factorizable via Fourier transform with respect to the $x_{-}$ coordinate:

$$
\begin{aligned}
& \delta\left(p_{+}-k_{+}-k_{1}^{+}-\cdots-k_{n}^{+}-z p_{+}\right) \\
& \quad=\int_{-\infty}^{+\infty} \frac{d x_{-}}{2 \pi} e^{i x_{-}\left(p_{+}(1-z)-k_{+}\right)} \prod_{i=1}^{n} e^{-i x_{-} k_{i}^{+}} .
\end{aligned}
$$

Therefore, to keep track of $(+)$ momentum-component conservation, it is enough to consider the $x_{-}$-dependent BFKL Green's function, which will satisfy the standard BFKL equation in rapidity space with the $e^{-i x_{-} k_{i}^{+}}$factor added to the real-emission term

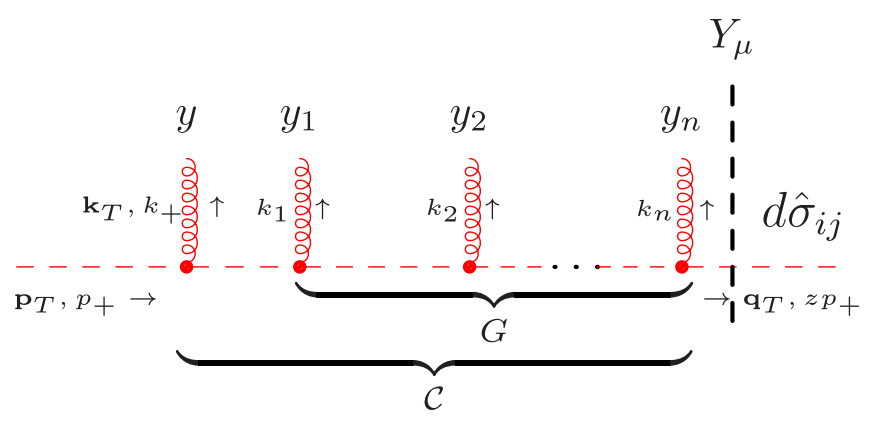

FIG. 1. Typical diagram for the amplitude corresponding to the resummation factor $\mathcal{C}$. The dashed line denotes the Reggeized gluon and solid circles are Lipatov's vertices. The rebounded gluon has transverse momentum $\mathbf{k}_{T}$ and rapidity $y$. Rapidities of all emitted gluons are ordered: $y>y_{1}>y_{2}>\ldots>y_{n}>Y_{\mu}$. The gluon with momentum $p^{+}$enters from a collinear PDF, and the nonzero $\mathbf{p}_{T}$ of this gluon is introduced to regularize collinear divergences (see the main text). The Reggeized gluon with transverse momentum $\mathbf{q}_{T}$ and $(+)$ momentum component $z p_{+}$ enters into the coefficient function $d \hat{\sigma}_{i j}$ of Eq. (1). 


$$
\begin{aligned}
G\left(\mathbf{q}_{T}^{2} \mid Y, \mathbf{p}_{T}^{2}, x_{-}\right)= & G_{0}\left(\mathbf{q}_{T}^{2} \mid \mathbf{p}_{T}^{2}, x_{-}\right)+\int_{0}^{Y} d y\left\{2 \omega_{g}\left(\mathbf{p}_{T}^{2}\right) G\left(\mathbf{q}_{T}^{2} \mid y, \mathbf{p}_{T}^{2}, x_{-}\right)\right. \\
& \left.+\hat{\alpha}_{s} \int \frac{d^{2-2 \epsilon} \mathbf{k}_{T}}{\pi(2 \pi)^{-2 \epsilon} \mathbf{k}_{T}^{2}} \exp \left[-i x_{-}\left|\mathbf{k}_{T}\right| e^{y}\right] G\left(\mathbf{q}_{T}^{2} \mid y,\left(\mathbf{p}_{T}-\mathbf{k}_{T}\right)^{2}, x_{-}\right)\right\}
\end{aligned}
$$

where we have introduced dimensional regularization with $\epsilon<0$ to regularize infrared divergences, $\omega_{g}\left(\mathbf{p}_{T}^{2}\right)$ is the one-loop Regge trajectory of a gluon

$$
\omega_{g}\left(\mathbf{p}_{T}^{2}\right)=-\frac{\hat{\alpha}_{s}}{4} \int \frac{d^{2-2 \epsilon} \mathbf{k}_{T}}{\pi(2 \pi)^{-2 \epsilon}} \frac{\mathbf{p}_{T}^{2}}{\mathbf{k}_{T}^{2}\left(\mathbf{p}_{T}-\mathbf{k}_{T}\right)^{2}}=\frac{\hat{\alpha}_{s}}{2 \epsilon}\left(\mathbf{p}_{T}^{2}\right)^{-\epsilon} \frac{(4 \pi)^{\epsilon} \Gamma(1+\epsilon) \Gamma^{2}(1-\epsilon)}{\Gamma(1-2 \epsilon)},
$$

and the initial condition $G_{0}$ is given by

$$
G_{0}\left(\mathbf{q}_{T}^{2} \mid \mathbf{p}_{T}^{2}, x_{-}\right)=\int_{0}^{+\infty} d q_{+} e^{-i x_{-} q_{+}} \delta\left(\frac{z p_{+}}{q_{+}}-1\right) \delta\left(\mathbf{q}_{T}^{2}-\mathbf{p}_{T}^{2}\right)=z p_{+} e^{-i z x_{-} p_{+}} \delta\left(\mathbf{q}_{T}^{2}-\mathbf{p}_{T}^{2}\right)
$$

Differentiating Eq. (7) with respect to $Y$, one obtains a differential form of the BFKL equation:

$$
\frac{\partial G\left(\mathbf{q}_{T}^{2} \mid Y, \mathbf{p}_{T}^{2}, x_{-}\right)}{\partial Y}=\hat{\alpha}_{s} \int d^{2-2 \epsilon} \mathbf{k}_{T} K\left(\mathbf{k}_{T}^{2}, \mathbf{p}_{T}^{2}, x_{-}, Y\right) G\left(\mathbf{q}_{T}^{2} \mid Y,\left(\mathbf{p}_{T}-\mathbf{k}_{T}\right)^{2}, x_{-}\right)
$$

with

$$
K\left(\mathbf{k}_{T}^{2}, \mathbf{p}_{T}^{2}, x_{-}, Y\right)=\delta^{(2-2 \epsilon)}\left(\mathbf{k}_{T}\right) \frac{\left(\mathbf{p}_{T}^{2}\right)^{-\epsilon}}{\epsilon} \frac{(4 \pi)^{\epsilon} \Gamma(1+\epsilon) \Gamma^{2}(1-\epsilon)}{\Gamma(1-2 \epsilon)}+\frac{\exp \left[-i x_{-}\left|\mathbf{k}_{T}\right| e^{Y}\right]}{\pi(2 \pi)^{-2 \epsilon} \mathbf{k}_{T}^{2}} .
$$

We use the fact that for $\mathbf{p}_{T} \neq 0$, the IR divergences cancel order by order in $\alpha_{s}$ in $G$, so one can safely introduce the Mellin transform for $\mathbf{p}_{T}^{2}$ dependence of the Green's function

$$
G\left(\mathbf{q}_{T}^{2} \mid Y, \mathbf{p}_{T}^{2}, x_{-}\right)=\int \frac{d \gamma}{2 \pi i} \frac{1}{\mathbf{p}_{T}^{2}}\left(\frac{\mathbf{p}_{T}^{2}}{\mu_{Y}^{2}}\right)^{\gamma} G\left(\mathbf{q}_{T}^{2} \mid Y, \gamma, x_{-}\right)
$$

and the kernel acts on powers of $\mathbf{p}_{T}^{2}$ as

$$
\lim _{\varepsilon \rightarrow 0} \int d^{2-2 \epsilon} \mathbf{k}_{T} K\left(\mathbf{k}_{T}^{2}, \mathbf{p}_{T}^{2}, x_{-}, Y\right)\left(\left(\mathbf{p}_{T}-\mathbf{k}_{T}\right)^{2}\right)^{-1+\gamma}=\left(\mathbf{p}_{T}^{2}\right)^{-1+\gamma} \tilde{\chi}\left(\gamma, x_{-}\left|\mathbf{p}_{T}\right| e^{Y}\right),
$$

with the function $\tilde{\chi}$ still depending on $\mathbf{p}_{T}$ through its argument $\tilde{x}_{-}=x_{-}\left|\mathbf{p}_{T}\right| e^{Y}$. Explicitly, this function is

$$
\begin{aligned}
\tilde{\chi}\left(\gamma, \tilde{x}_{-}\right) & =\lim _{\epsilon \rightarrow 0^{-}} \tilde{\chi}\left(\gamma, \tilde{x}_{-}, \epsilon\right) \\
& =\lim _{\epsilon \rightarrow 0^{-}}\left[\frac{1}{\epsilon} \frac{\Gamma(1+\epsilon) \Gamma^{2}(1-\epsilon)}{\Gamma(1-2 \epsilon)}+\int \frac{d^{2-2 \epsilon} \mathbf{l}_{T}}{\pi^{1-\epsilon}} \frac{\exp \left[-i \tilde{x}_{-}\left|\mathbf{l}_{T}\right|\right]}{\mathbf{l}_{T}^{2}\left(\left(\mathbf{n}_{T}-\mathbf{l}_{T}\right)^{2}\right)^{1-\gamma}}\right]
\end{aligned}
$$

where $\mathbf{n}_{T}$ is an arbitrary fixed unit vector in the transverse plane. One can write down an alternative definition of $\tilde{\chi}\left(\gamma, \tilde{x}_{-}\right)$ with infrared divergence canceled point by point in phase space (see again the textbook [53]). Using the explicit form of the Regge trajectory as an integral in transverse-momentum space (8) and the identity

$$
\frac{1}{\mathbf{l}_{T}^{2}\left(\mathbf{n}_{T}-\mathbf{l}_{T}\right)^{2}}=\frac{1}{\mathbf{l}_{T}^{2}\left[\mathbf{l}_{T}^{2}+\left(\mathbf{n}_{T}-\mathbf{l}_{T}\right)^{2}\right]}+\frac{1}{\left(\mathbf{n}_{T}-\mathbf{l}_{T}\right)^{2}\left[\mathbf{l}_{T}^{2}+\left(\mathbf{n}_{T}-\mathbf{l}_{T}\right)^{2}\right]},
$$

one obtains

$$
\tilde{\chi}\left(\gamma, \tilde{x}_{-}\right)=\int \frac{d^{2} \mathbf{l}_{T}}{\pi \mathbf{l}_{T}^{2}}\left[\left(\left(\mathbf{n}_{T}-\mathbf{l}_{T}\right)^{2}\right)^{\gamma-1} \exp \left[-i \tilde{x}_{-}\left|\mathbf{l}_{T}\right|\right]-\frac{1}{\mathbf{l}_{T}^{2}+\left(\mathbf{n}_{T}-\mathbf{l}_{T}\right)^{2}}\right]
$$


The latter definition is convenient for direct numerical evaluation of $\tilde{\chi}\left(\gamma, \tilde{x}_{-}\right)$. However, for analytic studies one would like to finally disentangle the $\mathbf{p}_{T}^{2}$ dependence from $\tilde{\chi}\left(\gamma, \tilde{x}_{-}\right)$. To this end, it is necessary to introduce the (inverse) Mellin transform over $\tilde{x}_{-}$as follows (see the Appendix for a detailed derivation):

$$
\tilde{\chi}\left(\gamma, \tilde{x}_{-}\right)=\chi(\gamma)+\int_{C_{\lambda}} \frac{d \lambda}{2 \pi i} \tilde{x}_{-}^{\lambda} \Delta \chi(\gamma, \lambda),
$$

where $\chi(\gamma)$ is an ordinary BFKL characteristic function (6), $\Delta \chi(\gamma, \lambda)$ is given by Eq. (A2) in the Appendix, and the contour $C_{\lambda}$ passes to the right from singularity of $\Delta \chi(\gamma, \lambda)$ at $\lambda=0$ parallel and in the same direction as the imaginary axis, thus,

$$
\lim _{\tilde{x}_{-} \rightarrow 0} \tilde{\chi}\left(\gamma, \tilde{x}_{-}\right)=\chi(\gamma)
$$

i.e., the limit $\tilde{x}_{-} \rightarrow 0$ corresponds to the usual BFKL evolution of the Green's function.

Using representation (15) and Eqs. (10)-(12), one obtains the evolution equation for $G\left(\mathbf{q}_{T}^{2} \mid Y, \gamma, x_{-}\right)$at arbitrary $x_{-}$:

$$
\begin{aligned}
\frac{\partial G\left(\mathbf{q}_{T}^{2} \mid Y, \gamma, x_{-}\right)}{\partial Y}= & \hat{\alpha}_{s} \chi(\gamma) G\left(\mathbf{q}_{T}^{2} \mid Y, \gamma_{,} x_{-}\right) \\
& +2 \hat{\alpha}_{s} \int_{C_{\lambda}^{\prime}} \frac{d \lambda}{2 \pi i}\left(\mu_{Y} x_{-} e^{Y}\right)^{2(\gamma-\lambda)} \\
& \times \Delta \chi(\lambda, 2(\gamma-\lambda)) G\left(\mathbf{q}_{T}^{2} \mid Y, \lambda, x_{-}\right),
\end{aligned}
$$

where the contour $C_{\lambda}^{\prime}$ goes in the positive direction of the imaginary axis of $\lambda$ and lies to the left of singularity of $\Delta \chi(\lambda, 2(\gamma-\lambda))$ at $\gamma=\lambda$ to be consistent with the definition of the contour in Eq. (15).

Since one has to do the inverse Fourier transform over $x_{-}$to finally obtain the UPDF via Eq. (4), then both small and large values of $x_{-}$will contribute. However, Eq. (17) is difficult to solve for general values of $x_{-}$, because it is still an integrodifferential equation. Because of Eq. (16), the limit of small $x_{-}$corresponds to ordinary BFKL evolution and reproduces the standard BFKL UPDF (5). In the present paper, we will concentrate on the opposite limit of large $x_{-}$. This limit is controlled by singularities of $\Delta \chi(\lambda, 2(\gamma-\lambda))$ to the right of the integration contour in Eq. (17), and hence, one should look for the pole at $\gamma-\lambda=0$ to obtain the leading-power contribution.

The detailed derivation in the Appendix shows that function $\Delta \chi(\gamma, \lambda)$ indeed has a pole at $\lambda=0$ :

$$
\Delta \chi(\gamma, \lambda)=-\frac{2}{\lambda^{2}}-\frac{1}{\lambda}\left(2 \gamma_{E}+i \pi+\chi(\gamma)\right)+O(1) .
$$

Before proceeding, it is instructive to understand the source of the $-2 / \lambda^{2}$ pole. From Eq. (14) one easily deduces the contribution of the region $\left|\mathbf{l}_{T}\right| \ll\left|\mathbf{n}_{T}\right|=1$ to $\tilde{\chi}\left(\gamma, \tilde{x}_{-}\right)$at $\tilde{x}_{-} \gg 1$ :

$$
\begin{aligned}
\tilde{\chi}\left(\gamma, \tilde{x}_{-}\right) & \simeq \int_{0}^{1} \frac{d \mathbf{l}_{T}^{2}}{\mathbf{l}_{T}^{2}}\left[\exp \left(-i \tilde{x}_{-}\left|\mathbf{l}_{T}\right|\right)-1\right] \\
& =-2 \ln \tilde{x}_{-}-2 \gamma_{E}-i \pi+O\left(\tilde{x}_{-}^{1}\right),
\end{aligned}
$$

and the term $-2 \ln \tilde{x}_{-}$corresponds to $-2 / \lambda^{2}$ pole in the $\lambda$ plane. From this short calculation, one can see that the double pole at $\lambda=0$ has infrared origin and both the realemission and Regge-trajectory terms of the BFKL kernel contribute to it.

Substituting Eq. (18) into (17) and taking the residue at $\lambda=\gamma$, one obtains

$$
\begin{aligned}
& {\left[\frac{\partial}{\partial Y}-\hat{\alpha}_{s} \frac{\partial}{\partial \gamma}\right] G\left(\mathbf{q}_{T}^{2} \mid Y, \gamma, x_{-}\right)} \\
& \quad=-\hat{\alpha}_{S} G\left(\mathbf{q}_{T}^{2} \mid Y, \gamma, x_{-}\right)\left[2 \gamma_{E}+i \pi+2 Y+2 \ln \left(\mu_{Y} x_{-}\right)\right] .
\end{aligned}
$$

Note that the characteristic function $\chi(\gamma)$ has canceled. This reflects a lack of nontrivial transverse-momentum dynamics in the Sudakov limit — a puzzling feature of this regime from the point of view of a physicist with a BFKL background.

The solution of this equation satisfying boundary condition (9) transferred from $\mathbf{p}_{T}^{2}$ to $\gamma$ space is

$$
\begin{aligned}
G\left(\mathbf{q}_{T}^{2} \mid Y, \gamma, x_{-}\right)= & z p_{+} e^{-i z x_{-} p_{+}}\left(\frac{\mathbf{q}_{T}^{2}}{\mu_{Y}^{2}}\right)^{-\gamma} \exp \left[-\hat{\alpha}_{S} Y\left(2 \gamma_{E}+i \pi\right.\right. \\
& \left.\left.+\ln \left(\mathbf{q}_{T}^{2} x_{-}^{2}\right)\right)-\hat{\alpha}_{S} Y^{2}\right] .
\end{aligned}
$$

In the present study, we will consider only leading effects due to the $\hat{\alpha}_{s} Y^{2}$ term in the exponent; therefore, in the next section we will use the simplified solution without subleading effects:

$$
G\left(\mathbf{q}_{T}^{2} \mid Y, \gamma, x_{-}\right)=z p_{+} e^{-i z x_{-} p_{+}}\left(\frac{\mathbf{q}_{T}^{2}}{\mu_{Y}^{2}}\right)^{-\gamma} e^{-\hat{\alpha}_{S} Y^{2}} .
$$

\section{SUDAKOV FORM FACTOR IN THE LLA}

Substituting the solution (21) as well as the inverse Mellin transform over $\gamma$ and Fourier transform over $x_{-}$into Eq. (4), one obtains

$$
\begin{aligned}
\tilde{\mathcal{C}}(z, & \left.\mathbf{q}_{T}^{2}, \mu_{Y} \mid \mathbf{p}_{T}^{2}\right) \\
= & \delta(z-1) \delta\left(\mathbf{q}_{T}^{2}-\mathbf{p}_{T}^{2}\right)+\hat{\alpha}_{s} \int \frac{d \gamma}{2 \pi i} \int_{0}^{\infty} d Y \int \frac{d \mathbf{k}_{T}^{2}}{\mathbf{k}_{T}^{2}} \\
& \times\left[\int_{-\infty}^{+\infty} \frac{d x_{-}}{2 \pi}\left(z p_{+}\right) \exp \left(i x_{-}\left(p_{+}(1-z)-\left|\mathbf{k}_{T}\right| e^{Y_{\mu}+Y}\right)\right)\right] \\
& \times \frac{1}{\left(\mathbf{p}_{T}-\mathbf{k}_{T}\right)^{2}}\left(\frac{\left(\mathbf{p}_{T}-\mathbf{k}_{T}\right)^{2}}{\mathbf{q}_{T}^{2}}\right)^{\gamma} e^{-\hat{\alpha}_{s} Y^{2}},
\end{aligned}
$$


where we have substituted $y=Y_{\mu}+Y$. The integral over $x_{-}$leads to the delta function, which can be used to integrate out $\mathbf{k}_{T}^{2}=p_{+}^{2}(1-z)^{2} e^{-2\left(Y_{\mu}+Y\right)}=$ $\mu_{Y}^{2}(1-z)^{2} e^{-2 Y} / z^{2}$, where we have used the relation
$Y_{\mu}=\ln \left(z p_{+}\right) / \mu_{Y}$. Because of the doubly logarithmic exponent, the rapidity integral will converge, and therefore, one can safely put $\mathbf{p}_{T}=0$ at this stage. Finally, one gets

$$
\tilde{\mathcal{C}}\left(z, \mathbf{q}_{T}^{2}, \mu_{Y} \mid \mathbf{p}_{T}^{2}=0\right)=\delta(z-1) \delta\left(\mathbf{q}_{T}^{2}\right)+\hat{\alpha}_{s} \frac{2 z^{3}}{\mu_{Y}^{2}(1-z)^{3}} \int \frac{d \gamma}{2 \pi i}\left(\frac{\mu_{Y}^{2}}{\mathbf{q}_{T}^{2}} \frac{(1-z)^{2}}{z^{2}}\right)^{\gamma} J\left(\gamma, \hat{\alpha}_{s}\right)
$$

where

$$
J(\gamma, \alpha)=\int_{0}^{\infty} d Y \exp \left[-\alpha Y^{2}+2 Y(1-\gamma)\right]=\frac{\sqrt{\pi}}{2 \sqrt{\alpha}} e^{\frac{(1-\gamma)^{2}}{\alpha}}\left[1+\operatorname{Erf}\left(\frac{1-\gamma}{\sqrt{\alpha}}\right)\right] .
$$

The latter function has the following expansion around $\alpha=0$ :

$$
J(\gamma, \alpha)=\sqrt{\frac{\pi}{\alpha}} e^{\frac{(1-\gamma)^{2}}{\alpha}}+\frac{1}{2(\gamma-1)}+\sum_{n=1}^{\infty} \frac{(2 n-1) !}{2^{2 n}(n-1) !} \frac{(-1)^{n} \alpha^{n}}{(\gamma-1)^{2 n+1}},
$$

so moving the Mellin contour in the $\gamma$ plane to the right one picks up the perturbative contribution of the essential singularity at $\gamma=1$, thus obtaining

$$
\tilde{\mathcal{C}}\left(z, \mathbf{q}_{T}^{2}, \mu_{Y} \mid \mathbf{p}_{T}^{2}=0\right)=\delta(z-1) \delta\left(\mathbf{q}_{T}^{2}\right)+\frac{\hat{\alpha}_{s}}{\mathbf{q}_{T}^{2}} \frac{z}{1-z} \exp \left[-\frac{\hat{\alpha}_{s}}{4} \ln ^{2}\left(\frac{\mu_{Y}^{2}}{\mathbf{q}_{T}^{2}} \frac{(1-z)^{2}}{z^{2}}\right)\right]
$$

We conclude that by picking up the leading $\hat{\alpha}_{s} Y^{2}$ term in the exponent of the BFKL Green's function (20), we have reproduced the well-known Sudakov doubly logarithmic suppression for the gluon unintegrated (or TMD) PDF at $\mathbf{q}_{T}^{2} \ll \mu_{Y}^{2}$ [14,55]. Equation (22) also features a doubly logarithmic suppression at $z \ll 1$, the significance of which requires further analysis. The second term in this equation has a subleading power in $z$ due to an overall factor of $z$ in front, while the standard BFKL result for UPDF (5) is of leading power in $z$. Equation (5) arises from consideration of small $x_{-} \ll \mu_{Y}^{-1}$, i.e., the BFKL limit of the solution of Eq. (17), while the NLL terms in the Sudakov regime arise from $\hat{\alpha}_{s} Y$ terms in the solution (20), which is valid at $x_{-} \gg \mu_{Y}^{-1}$.

\section{HEURISTIC DERIVATION}

A more intuitive derivation of the LL result for the Green's function (21) can be given as follows. From the lack of nontrivial $x_{-}$or $\gamma$ dependence of the doubly logarithmic solution (21), one concludes that in this approximation, transverse and longitudinal momenta of all real emissions on the stage of evolution of the Green's function are negligible in comparison to $\left|\mathbf{q}_{T}\right|$ and $\mu_{Y}$, respectively (Fig. 2). In the regime $\left|\mathbf{q}_{T}\right| \ll \mu_{Y}$, both of these conditions are satisfied by the following cut on the $(+)$ component of real-emission momentum

$$
k_{i}^{+}=\left|\mathbf{k}_{T i}\right| e^{y_{i}} \ll\left|\mathbf{q}_{T}\right|,
$$

where with logarithmic accuracy, one can replace $\ll \rightarrow<$ to obtain the following upper limit for the transverse momentum of real emission:

$$
\left|\mathbf{k}_{T i}\right|<\left|\mathbf{q}_{T}\right| e^{-y_{i}}
$$

This special kinematic situation is represented schematically in Fig. 2 and can be called the Sudakov cascade of emissions.

The cut (23) can be introduced to the well-known iterative solution of the BFKL equation (see, e.g., Ref. [56]) with explicit Regge factors $\exp \left[2 \omega_{g}\left(\mathbf{q}_{T i}\right)\left(y_{i+1}-y_{i}\right)\right]$ for each pair of adjacent emissions:

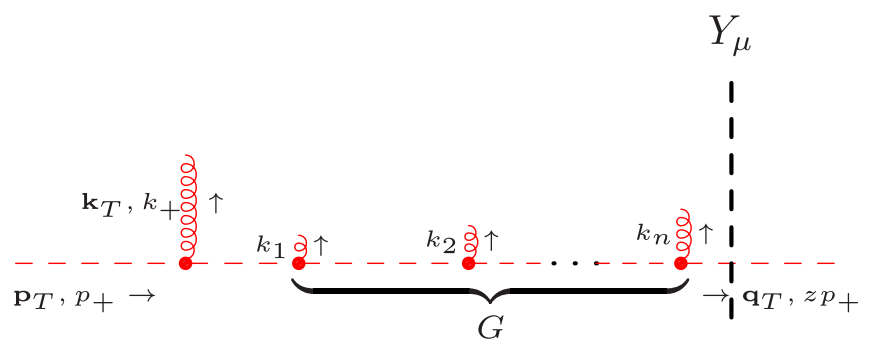

FIG. 2. The Sudakov cascade. The length of the gluon lines designates the magnitude of their transverse momenta. Rapidities of all emitted gluons are ordered: $y>y_{1}>y_{2}>\ldots>y_{n}>Y_{\mu}$. 


$$
\begin{aligned}
G\left(\mathbf{q}_{T}^{2} \mid Y, \mathbf{p}_{T}^{2}\right) & =\sum_{n=0}^{\infty} \frac{\hat{\alpha}_{s}^{n}}{\left[\pi(2 \pi)^{-2 \epsilon}\right]^{n}} \int \frac{d^{2-2 \epsilon} \mathbf{k}_{T 1}}{\mathbf{k}_{T 1}^{2}} \ldots \frac{d^{2-2 \epsilon} \mathbf{k}_{T n}}{\mathbf{k}_{T n}^{2}} \delta\left(\mathbf{p}_{T}-\mathbf{k}_{T 1}-\cdots-\mathbf{k}_{T n}-\mathbf{q}_{T}\right) \\
& \times \int_{0}^{Y} d y_{1} e^{2 \omega\left(\mathbf{p}_{T}\right) y_{1}} \int_{y_{1}}^{Y} d y_{2} e^{2 \omega\left(\mathbf{q}_{T 1}\right)\left(y_{2}-y_{1}\right)} \ldots \int_{y_{n-1}}^{Y} d y_{n} e^{2 \omega\left(\mathbf{q}_{T n}\right)\left(Y-y_{n-1}\right)},
\end{aligned}
$$

where $\mathbf{q}_{T i}=\mathbf{p}_{T}-\mathbf{k}_{T 1}-\cdots-\mathbf{k}_{T i}$.

Applying the cut (23) to Eq. (24), one obtains

$$
G\left(\mathbf{q}_{T}^{2} \mid Y, \mathbf{p}_{T}^{2}\right) \simeq \delta\left(\mathbf{p}_{T}-\mathbf{q}_{T}\right) e^{2 \omega_{g}\left(\mathbf{q}_{T}\right) Y} \sum_{n=0}^{\infty} \hat{\alpha}_{s}^{n} \int_{0}^{Y} d y_{1} \ldots \int_{y_{n-1}}^{Y} d y_{n} J_{\perp}\left(y_{1}\right) \ldots J_{\perp}\left(y_{n}\right)
$$

with

$$
J_{\perp}(y)=\int \frac{d^{2-2 \epsilon} \mathbf{k}_{T}}{\pi(2 \pi)^{-2 \epsilon} \mathbf{k}_{T}^{2}} \theta\left(\left|\mathbf{q}_{T}\right| e^{-y}-\left|\mathbf{k}_{T}\right|\right)=-\frac{1}{\epsilon} \frac{(4 \pi)^{\epsilon}}{\Gamma(1-\epsilon)}\left(\mathbf{q}_{T}^{2} e^{-2 y}\right)^{-\epsilon}=L_{\epsilon}-2 y+O(\epsilon),
$$

where $L_{\epsilon}=-1 / \epsilon+\ln \mathbf{q}_{T}^{2}+\gamma_{E}-\ln 4 \pi$, and one takes into account that the transverse momentum of each emission is negligible in comparison to $\mathbf{q}_{T}$, so that all $t$-channel transverse momenta are equal $\mathbf{q}_{T 1}=\ldots=\mathbf{q}_{T n}=\mathbf{p}_{T}=\mathbf{q}_{T}$ and all Regge factors cancel out except one: $\exp \left[2 \omega_{g}\left(\mathbf{q}_{T}\right) Y\right]$.

Up to $O(\epsilon)$, the sum in Eq. (25) leads to an exponent $\exp \left[\hat{\alpha}_{s} Y\left(L_{\epsilon}-Y\right)\right]$, because

$$
\int_{0}^{Y} d y_{1}\left(L_{\epsilon}-2 y_{1}\right) \int_{y_{1}}^{Y} d y_{2}\left(L_{\epsilon}-2 y_{2}\right) \ldots \int_{y_{n-1}}^{Y} d y_{n}\left(L_{\epsilon}-2 y_{n}\right)=\frac{1}{n !}\left[Y\left(L_{\epsilon}-Y\right)\right]^{n},
$$

and infrared divergence in $L_{\epsilon}$ cancels with the same divergence in the overall Regge factor; thus, one obtains

$$
G\left(\mathbf{q}_{T}^{2} \mid Y, \mathbf{p}_{T}^{2}\right) \simeq \delta\left(\mathbf{p}_{T}-\mathbf{q}_{T}\right) e^{-\hat{\alpha}_{s} Y^{2}}
$$

which coincides with Eq. (21). From Eq. (21), the final result for the doubly logarithmic Sudakov form factor (22) follows as described in Sec. IV.

In simple terms, the BFKL evolution has a natural tendency to produce emissions with substantial transverse and longitudinal momentum into a rapidity gap between the rebounded gluon and hard process; thus, the probability of "Sudakov cascade" configurations with no such emissions is suppressed by factor (21).

The physical argument above also exposes the relation between the Sudakov form factor in our calculation and Sudakov form factor in the Catani-Ciafaloni-FioraniMarcesini equation [57-60] and is closely related to parton-branching evolution equations [61-63]. In this approach, the form factor appears as the probability of no emissions between two "resolved" splittings. The angular ordering criterion for resolution of the splitting essentially reduces to rapidity ordering for small $\left|\mathbf{k}_{T}\right| \ll \mu_{Y}$ emissions. Thus, an alternative formulation of the abovementioned evolution equations is possible without an explicit Sudakov form factor, but in a form of BFKL-like evolution with longitudinal-momentum conservation. We leave this interesting subject for future studies.

\section{DISCUSSION AND CONCLUSIONS}

A few concluding remarks connecting our result with other studies in the literature are in order. First, it is important to clarify the role of conformal symmetry, which was an objective of Ref. [3]. One observes that if one has the solution $G\left(\mathbf{q}_{T}^{2} \mid Y, \mathbf{p}_{T}^{2}, x_{-}\right)$of Eq. (10), then $G\left(\mathbf{q}_{T}^{2} / \Lambda^{2} \mid Y, \mathbf{p}_{T}^{2} / \Lambda^{2}, \Lambda x_{-}\right)$with arbitrary rescaling parameter $\Lambda$ is also a solution. This rescaling symmetry may help to find a more convenient basis of functions for decomposition of the solution than Mellin representation (11) used in the present paper.

The appearance of variable $x_{-}$in our calculation and the separation of the $x_{-} \ll \mu_{Y}^{-1}$ (BFKL) and $x_{-} \gg \mu_{Y}^{-1}$ (Sudakov) regions clearly resembles the "thick shock-wave" picture of Refs. [1-3] and shows that from the rapidity factorization point of view, the Sudakov effects are the subset of subeikonal effects related to the longitudinal-momentum conservation and longitudinal structure of the target fields. It is interesting to connect this observation with recent developments in a field of subeikonal corrections in the high-energy limit; see, e.g., Refs. [64,65] and references therein.

The result (22) is also in agreement with the results of Ref. [11], where the consistency of the small- $x$ 
resummation with the Sudakov resummation in $\mathbf{x}_{T}$ space has been verified in one-loop approximation in examples of Higgs hadroproduction and heavy-quark photoproduction. For the case of Higgs hadroproduction, the term $-\alpha_{S} C_{A} /(2 \pi) \ln M_{H}^{2} \mathbf{x}_{T}^{2}$ was found at one loop, while for the case of heavy-quark photoproduction, the coefficient of the doubly logarithmic term was $-\alpha_{S} C_{A} /(4 \pi)$ as in our Eq. (22). The $\mathbf{x}_{T}$ space is convenient because the transverse-momentum convolution of two unintegrated PDFs turns into a product of their $\mathbf{x}_{T}$ images. Since for the Higgs hadroproduction one has two unintegrated PDFs for the initial state, the overall Sudakov form factor in $\mathbf{x}_{T}$ space is given by the product of two such form factors from each of the UPDFs. Hence, the coefficient of the double logarithm in $\mathbf{x}_{T}$ space is 2 times larger for the Higgs case than for the photoproduction case, which has only one UPDF in the initial state. Unfortunately, already at NLL level the structure of Sudakov logarithms for the production of colored final states becomes highly process dependent and cannot be attributed solely to the initial-state unintegrated or TMD PDFs. So HEF cannot reproduce all subleading logarithms in the Sudakov limit.

The derivation presented above brings hope to bridge the formal gap between high-energy QCD and TMD factorization by deriving some results of the latter from BFKL physics. This is a mathematically challenging task, because the solution of Eq. (17), which is valid in the whole range of $x_{-}$, has to be employed. But one might hope, as in the case of collinear anomalous dimension [54], that the BFKL derivation might provide some constraints for a higherorder structure of rapidity anomalous dimension, which governs the rapidity evolution of TMD PDFs, as well as for the structure of $\ln 1 / z$-enhanced corrections to collinear matching functions, which where recently found in the nextto-next-to-next-to-leading-order calculation of Ref. [66]. Another possible line of applications lies in the development of evolution equations based on the TMD splitting functions discovered in Refs. [16,67].

\section{ACKNOWLEDGMENTS}

The author acknowledges Ian Balitsky, Giovanni A. Chirilli, Francesco Hautmann, Jean-Philippe Lansberg, and other participants of the workshops of the series "Resummation Evolution Factorization" and "Quarkonia as Tools" for useful discussions, which have lead to the idea of this paper, as well as helpful referees for their comments. The work has been supported in part by the Ministry of Education and Science of Russia via state assignment to educational and research institutions under Project No. FSSS-2020-0014. The visit of M. A. N. to the IJClab has been supported by funding from the following sources: the European Union Horizon 2020 research and innovation program under the Grant Agreement No. 824093 in order to contribute to the EU Virtual Access "NLOAccess," the French ANR under Grant No. ANR-20-CE31-0015 ("PrecisOnium"), the Paris-Saclay U. via the P2I Department of the Physics Graduate School, the French CNRS via the "GDR QCD" via the IN2P3 project "GLUE@NLO" and via the IEA "GlueGraph" (Grant Agreement No. 205210), and the P2IO Labex via the Gluodynamics project.

\section{APPENDIX: THE CHARACTERISTIC FUNCTION}

The function $\Delta \chi(\gamma, \lambda)$ in Eq. (15) can be obtained from the $\epsilon \rightarrow 0$ limit of $\Delta \chi(\gamma, \lambda, \epsilon)$, which is given by the Mellin transform of the real-emission term of the Eq. (13):

$\Delta \chi(\gamma, \lambda, \epsilon)=\int_{0}^{\infty} d \tilde{x}_{-} \tilde{x}_{-}^{-1-\lambda} \int \frac{d^{2-2 \epsilon} \mathbf{l}_{T}}{\pi^{1-\epsilon}} \frac{\exp \left[-i \tilde{x}_{-}\left|\mathbf{l}_{T}\right|\right]}{\mathbf{l}_{T}^{2}\left(\left(\mathbf{n}_{T}-\mathbf{l}_{T}\right)^{2}\right)^{1-\gamma}}$.

After switching the order of integrations over $\tilde{x}_{-}$and $\mathbf{l}_{T}$, integration over $\tilde{x}_{-}$leads to a $\Gamma$ function, and $\mathbf{l}_{T}$ can be integrated out with the help of the formula

$$
\int \frac{d^{2-2 \epsilon} \mathbf{k}_{T}}{\left(\mathbf{k}_{T}^{2}\right)^{a}\left(\left(\mathbf{p}_{T}-\mathbf{k}_{T}\right)^{2}\right)^{b}}=\frac{\pi^{1-\epsilon}\left(\mathbf{p}_{T}^{2}\right)^{1-a-b-\epsilon} \Gamma(1-a-\epsilon) \Gamma(1-b-\epsilon) \Gamma(a+b+\epsilon-1)}{\Gamma(a) \Gamma(b) \Gamma(2-a-b-2 \epsilon)} ;
$$

thus, one obtains

$$
\Delta \chi(\gamma, \lambda, \epsilon)=e^{\frac{i \pi}{2} \lambda} \frac{\Gamma(-\lambda) \Gamma\left(\frac{\lambda}{2}-\epsilon\right) \Gamma(\gamma-\epsilon) \Gamma\left(1-\frac{\lambda}{2}-\gamma+\epsilon\right)}{\Gamma\left(1-\frac{\lambda}{2}\right) \Gamma(1-\gamma) \Gamma\left(\frac{\lambda}{2}+\gamma-2 \epsilon\right)} .
$$

At this stage, the integration contour in the $\lambda$ plane passes in between the leftmost pole of $\Gamma(-\lambda)$ at $\lambda=0$, and the rightmost pole of $\Gamma\left(\frac{\lambda}{2}-\epsilon\right)$ at $\lambda=-2 \epsilon$. In the limit $\epsilon \rightarrow 0^{-}$, these two poles pinch the contour to produce the infrared divergence of the real-emission term. To extract this divergence explicitly, one moves the contour to the right of the pole at $\lambda=0$, thus introducing the contour $C_{\lambda}$ used in Eq. (15) and picking up the residue at this pole. The sum of the residue of $\Delta \chi(\gamma, \lambda, \epsilon)$ at $\lambda=0$ and the Regge-trajectory contribution in Eq. (13) gives the $\chi(\gamma)$ term in Eq. (15):

$$
\lim _{\epsilon \rightarrow 0^{-}}\left[-\underset{\lambda=0}{\operatorname{res} \Delta \chi}(\gamma, \lambda, \epsilon)+\frac{1}{\epsilon} \frac{\Gamma(1+\epsilon) \Gamma^{2}(1-\epsilon)}{\Gamma(1-2 \epsilon)}\right]=\chi(\gamma) .
$$

After moving the contour past the $\lambda=0$ pole, there is no pinch, and one can safely put $\epsilon=0$, leading to the $\Delta \chi(\gamma, \lambda)$ function to be used in Eq. (15): 


$$
\Delta \chi(\gamma, \lambda)=\lim _{\epsilon \rightarrow 0^{-}} \Delta \chi(\gamma, \lambda, \epsilon)=e^{\frac{i \pi}{2} \lambda} \frac{\Gamma(-\lambda) \Gamma\left(\frac{\lambda}{2}\right) \Gamma(\gamma) \Gamma\left(1-\frac{\lambda}{2}-\gamma\right)}{\Gamma\left(1-\frac{\lambda}{2}\right) \Gamma(1-\gamma) \Gamma\left(\frac{\lambda}{2}+\gamma\right)} .
$$

Expansion of this function around $\lambda=0$ is given in Eq. (18).

Summing up residues in the poles at $\lambda=n$ with integer $n>0$ and $\lambda=2(2 n+2-\gamma)$ with $n=0,1,2, \ldots$ in Eq. (15), one obtains the following exact expression for the $\tilde{x}_{-}$-dependent characteristic function in terms of known hypergeometric functions:

$$
\begin{aligned}
\tilde{\chi}_{-}\left(\gamma, \tilde{x}_{-}\right)= & \chi(\gamma)-i \tilde{x}_{-} \frac{\Gamma\left(\frac{1}{2}-\gamma\right) \Gamma(\gamma)}{\Gamma(1-\gamma) \Gamma\left(\frac{1}{2}+\gamma\right)}{ }_{1 / 2} F_{1 / 2}\left(\frac{3}{2}, \frac{1}{2}+\gamma, \frac{1}{2}+\gamma \mid-\frac{\tilde{x}_{-}^{2}}{4}\right) \\
& -2 e^{-i \pi \gamma} \tilde{x}_{-}^{2-2 \gamma} \Gamma(2 \gamma-2)_{1-\gamma} F_{1-\gamma}\left(1, \frac{3}{2}-\gamma, 2-\gamma \mid-\frac{\tilde{x}_{-}^{2}}{4}\right) .
\end{aligned}
$$

This expression has been cross-checked against direct numerical evaluation of Eq. (14). At $\left|\tilde{x}_{-}\right| \gg 1$, Eq. (A3) has the asymptotics:

$$
\tilde{\chi}\left(\gamma, \tilde{x}_{-}\right)=-2 \gamma_{E}-i \pi-2 \ln \tilde{x}_{-}+\tilde{x}_{-}^{-2 \gamma} e^{-i \tilde{x}_{-}} \frac{2 \cos (\pi \gamma) \Gamma(2 \gamma) \Gamma\left(\frac{1}{2}-\gamma\right)}{\sqrt{\pi} \Gamma(1-\gamma)}+O\left(\tilde{x}_{-}^{-1}\right)
$$

From the point of view of integral representation (15), the non-power-suppressed part of the asymptotics (A4) arises due to the pole at $\lambda=0$ to the left of the contour $C_{\lambda}$, while the $O\left(\tilde{x}_{-}^{2 \gamma}\right)$-suppressed term is due to the contribution of essential singularity at $\lambda=\infty$. The essential singularity contributes because of the exp $[i \pi \lambda / 2]$ factor in Eq. (A2), which cancels the exponential damping due to the $\Gamma$ functions in the quadrant $\operatorname{Im} \lambda<0$ and $\operatorname{Re} \lambda<0$. Strictly speaking, this behavior of $\Delta \chi(\gamma, \lambda)$ at $\lambda \rightarrow \infty$ prevents one form closing of the contour $C_{\lambda}$ around the poles to the left of it; hence, representation (A3) has been obtained by closing of the contour around the right-hand poles, which is allowed by Jordan's lemma. However, as one can see from Eq. (A4), the contribution of the pole at $\lambda=0$ has correctly reproduced non-power-suppressed terms in the asymptotics of Eq. (A3), and therefore, our calculation after Eq. (17) is correct.

The modified characteristic function $\tilde{\chi}\left(\gamma, \tilde{x}_{-}\right)$is plotted as a function of $\gamma$ in Fig. 3. It reduces to Lipatov's one (6) in the limit $\tilde{x}_{-} \rightarrow 0$, but the so-called "anticollinear" pole at $\gamma=1$ is very sensitive to $\tilde{x}_{-}$and disappears for any nonzero value of it. The "collinear" pole at $\gamma=0$ stays intact, which is to be expected, because it is important for proper factorization of collinear divergences from the UPDF. The $\tilde{x}_{-}$dependence is
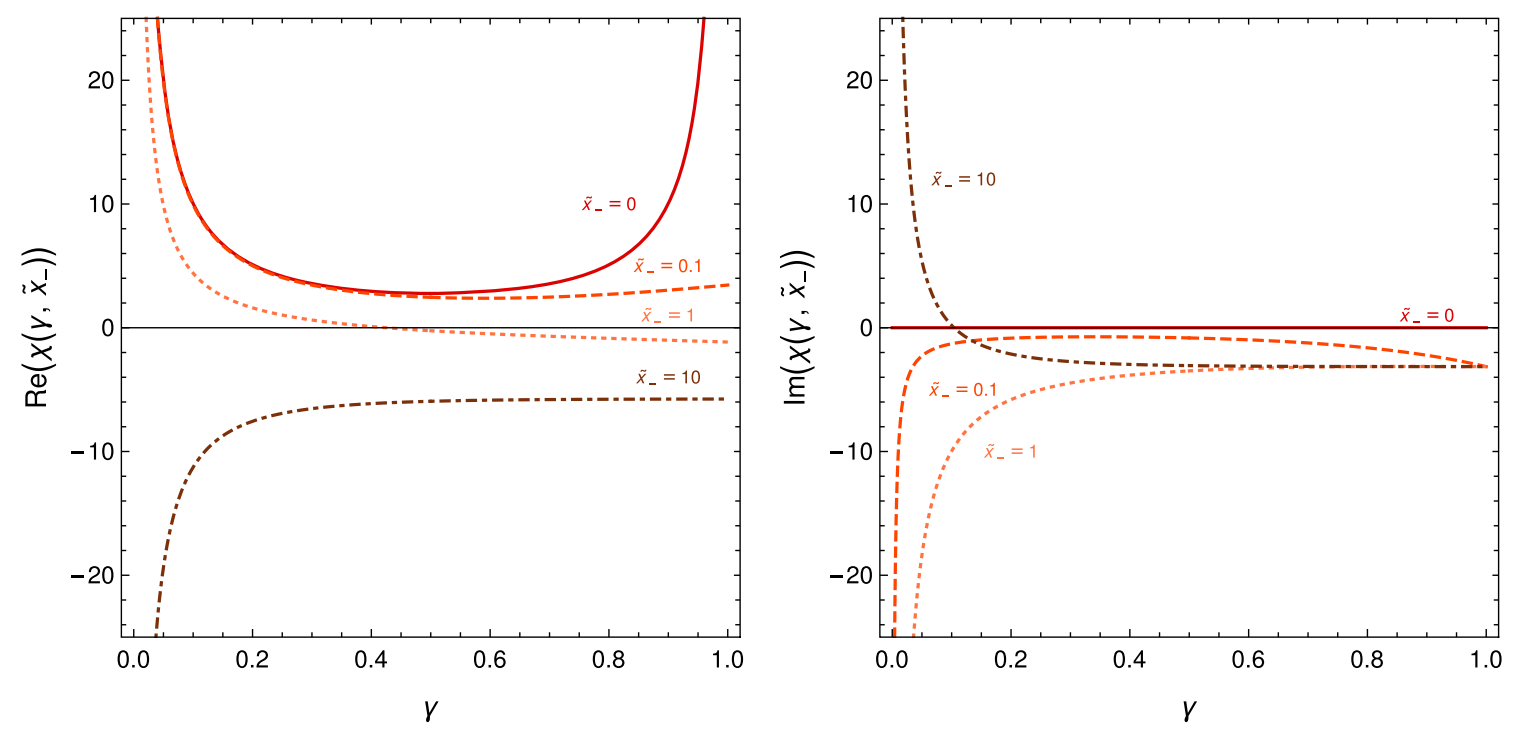

FIG. 3. The $\gamma$ dependence of the real (left panel) and imaginary (right panel) parts of the $\tilde{x}_{-}$-dependent characteristic function (13) for $\tilde{x}_{-}=0$ (solid line), 0.1 (dashed line), 1 (dotted line), and 10 (dash-dotted line). 

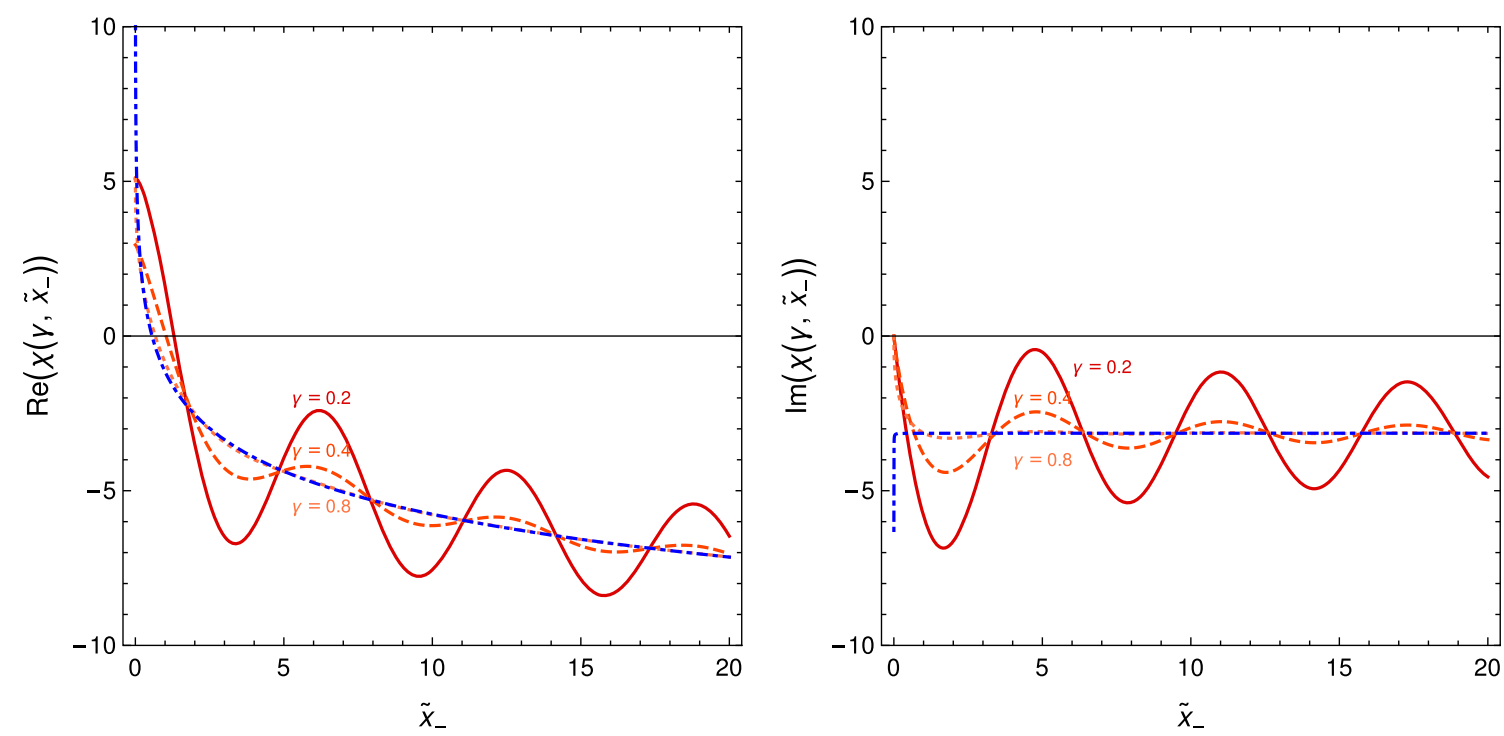

FIG. 4. The $\tilde{x}_{-}$dependence of the real (left panel) and imaginary (right panel) parts of the characteristic function (13) for $\gamma=0.2$ (solid line), 0.4 (dashed line), and 0.8 (dotted line). The non-power-suppressed part of asymptotic expression (A4) is shown by the dash-dotted line.

shown in Fig. 4 for $\tilde{x}_{-}>0$. Dependence for $\tilde{x}_{-}<0$ follows from the fact that for real $\tilde{x}_{-}$and $\gamma$, one has

$$
\tilde{\chi}\left(\gamma,-\tilde{x}_{-}\right)=\tilde{\chi}^{*}\left(\gamma, \tilde{x}_{-}\right)
$$

due to definition (13). The asymptotics (A4) is satisfied, and we emphasize that the LL Sudakov form factor follows essentially from the presence of the $\ln \tilde{x}_{-}$term in this asymptotic expression.
[1] I. Balitsky and A. Tarasov, Rapidity evolution of gluon TMD from low to moderate $x$, J. High Energy Phys. 10 (2015) 017.

[2] I. Balitsky and A. Tarasov, Gluon TMD in particle production from low to moderate $x$, J. High Energy Phys. 06 (2016) 164.

[3] I. Balitsky and G. A. Chirilli, Conformal invariance of transverse-momentum dependent parton distributions rapidity evolution, Phys. Rev. D 100, 051504 (2019).

[4] P. Kotko, K. Kutak, C. Marquet, E. Petreska, S. Sapeta, and A. van Hameren, Improved TMD factorization for forward dijet production in dilute-dense hadronic collisions, J. High Energy Phys. 09 (2015) 106.

[5] T. Altinoluk and R. Boussarie, Low $x$ physics as an infinite twist (G)TMD framework: Unravelling the origins of saturation, J. High Energy Phys. 10 (2019) 208.

[6] T. Altinoluk, R. Boussarie, and P. Kotko, Interplay of the CGC and TMD frameworks to all orders in kinematic twist, J. High Energy Phys. 05 (2019) 156.

[7] T. Altinoluk, R. Boussarie, C. Marquet, and P. Taels, Photoproduction of three jets in the CGC: Gluon TMDs and dilute limit, J. High Energy Phys. 07 (2020) 143.

[8] R. Boussarie and Y. Mehtar-Tani, A novel formulation of the unintegrated gluon distribution for DIS, arXiv:2006.14569.
[9] B.-W. Xiao and F. Yuan, BFKL and Sudakov resummation in Higgs boson plus jet production with large rapidity separation, Phys. Lett. B 782, 28 (2018).

[10] A. H. Mueller, L. Szymanowski, S. Wallon, B.-W. Xiao, and F. Yuan, Sudakov resummations in Mueller-Navelet dijet production, J. High Energy Phys. 03 (2016) 096.

[11] A. H. Mueller, B.-W. Xiao, and F. Yuan, Sudakov double logarithms resummation in hard processes in the small- $x$ saturation formalism, Phys. Rev. D 88, 114010 (2013).

[12] I. Balitsky, Operator expansion for high-energy scattering, Nucl. Phys. B463, 99 (1996).

[13] Y. V. Kovchegov, Small $x F(2)$ structure function of a nucleus including multiple pomeron exchanges, Phys. Rev. D 60, 034008 (1999).

[14] J.C. Collins, Foundations of Perturbative $Q C D$ (Cambridge University Press, Cambridge, England, 2011).

[15] From this point of view, it is interesting to study the relation between real-emission kernel of Refs. [1,2] and TMD gluon-gluon splitting function derived in Ref. [16].

[16] M. Hentschinski, A. Kusina, K. Kutak, and M. Serino, TMD splitting functions in $k_{T}$ factorization: The real contribution to the gluon-to-gluon splitting, Eur. Phys. J. C 78, 174 (2018). 
[17] E. A. Kuraev, L. N. Lipatov, and V. S. Fadin, Multi-Reggeon processes in the Yang-Mills theory, Sov. Phys. JETP 44, 443 (1976).

[18] E. A. Kuraev, L. N. Lipatov, and V. S. Fadin, The Pomeranchuk singularity in non-Abelian gauge theories, Sov. Phys. JETP 45, 199 (1977).

[19] Ya. Ya. Balitsky and L. N. Lipatov, The Pomeranchuk singularity in quantum chromodynamics, Sov. J. Nucl. Phys. 28, 822 (1978).

[20] T. Becher and G. Bell, Analytic regularization in softcollinear effective theory, Phys. Lett. B 713, 41 (2012).

[21] T. Becher, M. Neubert, and D. Wilhelm, Electroweak gaugeboson production at small $q_{t}$ : Infrared safety from the collinear anomaly, J. High Energy Phys. 02 (2012) 124.

[22] J.-Yu Chiu, A. Jain, D. Neill, and I. Z. Rothstein, A formalism for the systematic treatment of rapidity logarithms in quantum field theory, J. High Energy Phys. 05 (2012) 084.

[23] L. N. Lipatov, Gauge invariant effective action for high-energy processes in QCD, Nucl. Phys. B452, 369 (1995).

[24] J. Bartels, L. N. Lipatov, and G. P. Vacca, Ward identities for amplitudes with Reggeized gluons, Phys. Rev. D 86, 105045 (2012).

[25] G. Chachamis, M. Hentschinski, J. D. Madrigal Martinez, and A. Sabio Vera, Next-to-leading order corrections to the gluon-induced forward jet vertex from the high energy effective action, Phys. Rev. D 87, 076009 (2013).

[26] G. Chachamis, M. Hentschinski, J. D. Madrigal Martinez, and A. Sabio Vera, Quark contribution to the gluon Regge trajectory at NLO from the high energy effective action, Nucl. Phys. B861, 133 (2012).

[27] G. Chachamis, M. Hentschinski, J. D. Madrigal Martinez, and A. Sabio Vera, Gluon Regge trajectory at two loops from Lipatov's high energy effective action, Nucl. Phys. B876, 453 (2013).

[28] I. Z. Rothstein and I. W. Stewart, An effective field theory for forward scattering and factorization violation, J. High Energy Phys. 08 (2016) 025.

[29] I. Moult, M. P. Solon, I. W. Stewart, and G. Vita, Fermionic glauber operators and quark Reggeization, J. High Energy Phys. 02 (2018) 134.

[30] M. A. Kimber, A. D. Martin, and M. G. Ryskin, Unintegrated parton distributions, Phys. Rev. D 63, 114027 (2001).

[31] G. Watt, A. D. Martin, and M. G. Ryskin, Unintegrated parton distributions and inclusive jet production at HERA, Eur. Phys. J. C 31, 73 (2003).

[32] G. Watt, A. D. Martin, and M. G. Ryskin, Unintegrated parton distributions and electroweak boson production at hadron colliders, Phys. Rev. D 70, 014012 (2004); Erratum, Phys. Rev. D 70, 079902 (2004).

[33] A. D. Martin, M. G. Ryskin, and G. Watt, NLO prescription for unintegrated parton distributions, Eur. Phys. J. C 66, 163 (2010).

[34] M. A. Nefedov, V. A. Saleev, and A. V. Shipilova, Dijet azimuthal decorrelations at the LHC in the parton Reggeization approach, Phys. Rev. D 87, 094030 (2013).

[35] M. Bury, M. Deak, K. Kutak, and S. Sapeta, Single and double inclusive forward jet production at the LHC at $\sqrt{s}=$ 7 and 13 TeV, Phys. Lett. B 760, 594 (2016).
[36] A. van Hameren, P. Kotko, K. Kutak, and S. Sapeta, Sudakov effects in central-forward dijet production in high energy factorization, Phys. Lett. B 814, 136078 (2021).

[37] K. Kutak, R. Maciula, M. Serino, A. Szczurek, and A. van Hameren, Four-jet production in single- and double-parton scattering within high-energy factorization, J. High Energy Phys. 04 (2016) 175.

[38] A. V. Karpishkov, M. A. Nefedov, and V. A. Saleev, $B \bar{B}$ angular correlations at the LHC in parton Reggeization approach merged with higher-order matrix elements, Phys. Rev. D 96, 096019 (2017).

[39] R. Maciula and A. Szczurek, Consistent treatment of charm production in higher-orders at tree-level within $k_{T^{-}}$ factorization approach, Phys. Rev. D 100, 054001 (2019).

[40] R. Maciula and A. Szczurek, Consistent treatment of charm production in higher-orders at tree-level within $k_{T}$-factorization approach, Phys. Rev. D 100, 054001 (2019).

[41] Z.-G. He, B. A. Kniehl, M. A. Nefedov, and V. A. Saleev, Double Prompt $J / \psi$ Hadroproduction in the Parton Reggeization Approach with High-Energy Resummation, Phys. Rev. Lett. 123, 162002 (2019).

[42] M. A. Nefedov, N. N. Nikolaev, and V. A. Saleev, Drell-Yan lepton pair production at high energies in the parton Reggeization approach, Phys. Rev. D 87, 014022 (2013).

[43] M. Nefedov and V. Saleev, Off-shell initial state effects, gauge invariance and angular distributions in the Drell-Yan process, Phys. Lett. B 790, 551 (2019).

[44] M. A. Nefedov and V. A. Saleev, High-energy factorization for Drell-Yan process in $p p$ and $p \bar{p}$ collisions with new unintegrated PDFs, Phys. Rev. D 102, 114018 (2020).

[45] S. Catani, M. Ciafaloni, and F. Hautmann, Gluon contributions to small $x$ heavy flavor production, Phys. Lett. B 242, 97 (1990).

[46] S. Catani, M. Ciafaloni, and F. Hautmann, High-energy factorization and small $x$ heavy flavor production, Nucl. Phys. B366, 135 (1991).

[47] J. C. Collins and R. Keith Ellis, Heavy quark production in very high-energy hadron collisions, Nucl. Phys. B360, 3 (1991).

[48] S. Catani and F. Hautmann, High-energy factorization and small $x$ deep inelastic scattering beyond leading order, Nucl. Phys. B427, 475 (1994).

[49] R. D. Ball, V. Bertone, M. Bonvini, S. Marzani, J. Rojo, and L. Rottoli, Parton distributions with small- $x$ resummation: Evidence for BFKL dynamics in HERA data, Eur. Phys. J. C 78, 321 (2018).

[50] H. Abdolmaleki et al. (xFitter Developers' Team), Impact of low- $x$ resummation on QCD analysis of HERA data, Eur. Phys. J. C 78, 621 (2018).

[51] F. Hautmann, Heavy top limit and double logarithmic contributions to Higgs production at $\left(m_{H^{2}} / s \ll 1\right)$, Phys. Lett. B 535, 159 (2002).

[52] R. V. Harlander, H. Mantler, S. Marzani, and K. J. Ozeren, Higgs production in gluon fusion at next-to-next-to-leading order QCD for finite top mass, Eur. Phys. J. C 66, 359 (2010).

[53] Y. V. Kovchegov and E. Levin, Quantum Chromodynamics at High Energy, Cambridge Monographs on Particle Physics, Nuclear Physics and Cosmology (Cambridge University Press, Cambridge, England, 2012). 
[54] T. Jaroszewicz, Gluonic Regge singularities and anomalous dimensions in QCD, Phys. Lett. 116B, 291 (1982).

[55] Y. L. Dokshitzer, D. Diakonov, and S. I. Troian, Hard processes in quantum chromodynamics, Phys. Rep. 58, 269 (1980).

[56] C. R. Schmidt, A Monte Carlo Solution to the BFKL Equation, Phys. Rev. Lett. 78, 4531 (1997).

[57] M. Ciafaloni, Coherence effects in initial jets at small $\left(q^{2} / s\right)$, Nucl. Phys. B296, 49 (1988).

[58] S. Catani, F. Fiorani, and G. Marchesini, Small $x$ behavior of initial state radiation in perturbative QCD, Nucl. Phys. B336, 18 (1990).

[59] S. Catani, F. Fiorani, and G. Marchesini, QCD coherence in initial state radiation, Phys. Lett. B 234, 339 (1990).

[60] G. Marchesini, QCD coherence in the structure function and associated distributions at small $x$, Nucl. Phys. B445, 49 (1995).

[61] A. Bermudez Martinez, P. Connor, H. Jung, A. Lelek, R. lebk, F. Hautmann, and V. Radescu, Collinear and TMD parton densities from fits to precision DIS measurements in the parton branching method, Phys. Rev. D 99, 074008 (2019).
[62] F. Hautmann, L. Keersmaekers, A. Lelek, and A. M. Van Kampen, Dynamical resolution scale in transverse momentum distributions at the LHC, Nucl. Phys. B949, 114795 (2019).

[63] A. Bermudez Martinez, F. Hautmann, and M. L. Mangano, TMD evolution and multi-jet merging, arXiv:2107 .01224 .

[64] G. Antonio Chirilli, Sub-eikonal corrections to scattering amplitudes at high energy, J. High Energy Phys. 01 (2019) 118.

[65] P. Agostini, T. Altinoluk, and N. Armesto, Non-eikonal corrections to multi-particle production in the color glass condensate, Eur. Phys. J. C 79, 600 (2019).

[66] M.-x. Luo, T.-Z. Yang, H. Xing Zhu, and Y. J. Zhu, Quark Transverse Parton Distribution at the Next-toNext-to-Next-to-Leading Order, Phys. Rev. Lett. 124, 092001 (2020).

[67] O. Gituliar, M. Hentschinski, and K. Kutak, Transversemomentum-dependent quark splitting functions in $k_{T^{-}}$ factorization: Real contributions, J. High Energy Phys. 01 (2016) 181. 